T-jump and circular dichroism: folding dynamics in proteins and DNA

Pascale Changenet and François Hache*

Laboratoire d'optique et Biosciences, Ecole Polytechnique, CNRS, INSERM, IP Paris, 91128 Palaiseau cedex, France

Francois.hache@polytechnique.edu 


\title{
T-jump and circular dichroism: folding dynamics in proteins and DNA
}

\author{
We review recent experimental work combining circular dichroism spectroscopy \\ and T-jump experiment to investigate elementary dynamics in the \\ folding/unfolding of biomolecules. A nanosecond set-up is presented for \\ poly(Glutamic acid) studies whereas a millisecond one is developed for \\ investigation of DNA G-quadruplex dynamics.
}

Keywords: circular dichroism; T-jump, protein folding; DNA G-quadruplex

\section{Introduction}

Investigating the ultrafast dynamics of folding/unfolding of biological scaffolds such as protein secondary structures or nucleic acid conformations is of primary importance to gain insight into the kinetics and the energetics of these structures[1]. Many physiological or pathophysiological processes rely on those processes and only few information is available on the fundamental mechanisms.

Ultrafast absorption and fluorescence spectroscopy offers many possibilities to investigate rapid processes in molecules and extension to the deep-UV has been implemented recently for biomolecules [2]. Such techniques are however not adequate to study the molecular conformations or are limited to very specific samples and usually require chemical modifications of the molecules under study. By contrast, circular dichroism spectroscopy is a sensitive probe of the conformation of molecules in their natural state that does not require any modification [3]. In particular, proteins and DNAs have characteristic bands lying in the middle-UV which are closely connected to their spatial conformation.

Triggering of folding/unfolding in biomolecules is another issue which deserves special attention [4]. Here again, a very nice opportunity to provoke conformational changes in biomolecules in their natural state, without any perturbation, is to perform T- 
jump experiments [5]. In these experiments, the solvent (usually water) surrounding the biomolecules is rapidly heated up by a laser pulse. This temperature change shifts the thermodynamic equilibrium and induces an evolution of the biomolecules towards this new state which can be monitored by UV circular dichroism and absorption.

In this article, we review our recent experiments carried out on the formation dynamics of two different systems: $\alpha$-helices in polypeptides and DNA Gquadruplexes. Their temporal behaviour has been found to be quite different but thanks to specific experimental developments, relevant timescales could be measured in each case. These experiments demonstrate the versatility of the T-jump/CD combination to investigate elementary processes in biological samples under near physiological conditions.

\section{Secondary structure dynamics in proteins: nanosecond $\mathbf{T}-\mathbf{j u m p}$}

3D structure in proteins plays an outstanding role in physiology. Proper functioning of the enzymes rely on their correct folding and conversely, improper folding can result in severe diseases such as Alzheimer or Parkinson ones [6]. The most rapid secondary structure that forms in proteins is $\alpha$-helix and much work has been devoted to its dynamical properties [7]. Here, we choose to investigate the folding dynamics of a model polypeptide, poly(Glutamic acid), whose propensity to form $\alpha$-helix is very high. The originality of our approach relies on the possibility to study of this sample in natural water in order to stick to the physiological conditions, in contrast to frequentlyused IR studies which probe the Amide I' band and require to use heavy water .

\section{Experimental set-up}

The expected timescale of poly(Glutamic acid) folding being in the microsecond range, 
we have developed a standard nanosecond T-jump experiment (Fig. 1a). Thanks to a nanosecond OPO pumped by a $20 \mathrm{~Hz} \mathrm{Nd:YAG} \mathrm{laser,} \mathrm{we} \mathrm{generated} 5 \mathrm{~ns}, 6 \mathrm{~mJ}$ pulses at $1.5 \mu \mathrm{m}$ in order to excite the $v_{2}+v_{3}$ overtone of water vibration to instantaneously heat up a $100 \mu \mathrm{m}$-thick sample silica cell. The cell was thermalized by an external water circulation. Monitoring of the temperature jump and its dynamics in situ were achieved with a Bromothymol blue solution. Temperature changes up to $8^{\circ} \mathrm{C}$ can be reached. Heating time was found to be below the $5 \mathrm{~ns}$ resolution of our set-up. The temperature remains then constant for about $800 \mu$ s and goes back to the initial temperature within a few milliseconds [8].

\section{Experiments in poly(Glutamic acid)}

We studied a polymer of Glutamic acid (Sigma-Aldrich) dissolved without further purification in water $(20 \mathrm{mg} / \mathrm{ml})$. $\mathrm{pH}$ between 4.2 and 5.4 were considered: at such $\mathrm{pH}$, the peptide is known to form an $\alpha$-helix that is ascertained by its characteristic CD signal at $220 \mathrm{~nm}$. Fig. 2 represents the evolution of the CD spectra as a function of the temperature, denoting the thermal unfolding occurring at high temperatures.

In order to monitor the dynamics of the peptide unfolding, we measured the absorption and the CD changes at 220nm triggered by T-jump. To reach this frequency domain, we performed two stages of second-harmonic generation of a quasi-cw Titanium-sapphire laser to generate the fourth harmonic of $880 \mathrm{~nm}$ pulses. Polarization of these middle-UV pulses are then alternately right and left circularly-polarized by a Pockels cell. Monitoring of the transmitted intensity by a photomultiplier tube and a rapid oscilloscope allowed to extract together the change in absorption and in $\mathrm{CD}$ with a time resolution down to about $100 \mathrm{~ns}$. 
T-jump/CD measurements carried out for several $\mathrm{pH}$ are depicted in fig. 3 . The observed decrease in absorption as well as in CD corresponds to the unfolding of the peptide following the temperature jump due to the change of the thermodynamic conditions. Relaxation times in the $\mu$ s range are readily obtained from these curves, yielding the effective transition rate of the reaction.

\section{Discussion}

Under the assumption that the peptide is at thermodynamic equilibrium between a folded and an unfolded states $P G A_{\text {unfolded }} \underset{k_{u}}{\stackrel{k_{f}}{\leftarrow}} P G A_{\text {folded }}$, the experimental relaxation rate is actually the sum of the two basic processes: $k_{o b s}=k_{f}+k_{u}$. It is thus possible to extract independently $k_{f}$ and $k_{u}$ by considering the evolution of the steady-state CD signal as function of the temperature. Details can be found in Ref. [9]. Finally, by plotting the Arrhenius curves for both rates, one can deduce the energetic parameters of the peptide: the folding/unfolding reaction enthalpy $\left(\Delta H_{m}\right)$ and the folding $\left(E_{a, f}\right)$ and unfolding $\left(E_{a u}\right)$ activation energies. Schematic of the energetic profile of the reaction is depicted in fig. 4 and numerical values of the various parameters obtained from the experiments are given in Table 1. The markedly increase of the activation energies with the $\mathrm{pH}$ decrease is consistent with the side chain of the Glutamate residues becoming protonated and uncharged, favoring the peptide folding[10].

\section{G-quadruplex folding dynamics: millisecond T-jump}

G-quadruplexes (G4) are non-canonical DNA structures, which result from the 
hydrophobic stacking of a number of guanine quartets stabilized by metal cations. G4 are highly polymorphic structures that are involved in important cellular regulation functions. They are present in particular in human telomeres and in oncogene promoters and are considered potential targets for anticancerous strategies [11]. G4 provide a large set of topologies particularly attractive for the fundamental studies of DNA folding processes. However, despite recent experimental and theoretical efforts [12], there is no consensual picture of their folding mechanisms. We have therefore investigated a 21 mer, 5'-d[GGG(GGGTTA) 3]-3' (Tel21), which represents the shortest human telomeric sequence [13], known to mainly assume an antiparallel topology in the presence of $\mathrm{Na}+$, giving rise to a characteristic CD peak centred around $293 \mathrm{~nm}$ (Fig. 5).

\section{Experimental set-up}

First experiments performed with our previously described set-up provided evidence that folding/unfolding dynamics of G4 structures involves timescales much longer than the millisecond range. We have therefore come up with another T-jump set-up where water heating is achieved with a $5 \mathrm{~W}$ cw laser diode at $1.5 \mu \mathrm{m}$. With this device, temperature jump is achieved in a few tens of milliseconds and remains constant as long as the laser is on. After switching off the laser, the temperature comes back to its initial value in a few tens of milliseconds. The experiments consist of two steps: heating up of the sample during $4 \mathrm{~s}$ followed by cooling down for $5 \mathrm{~s}$ [14].

Monitoring of the G4 conformation was achieved through time-resolved CD measurement at $293 \mathrm{~nm}$ produced from the third harmonic $(\omega+2 \omega)$ of the quasi-cw titanium-sapphire laser (Fig. 1b). The sample was placed in a home-made $\mathrm{CaF}_{2}$ cell, insuring a better temperature homogeneity than silica cells. In order to measure the CD 
signals, a photoelastic modulator $(50 \mathrm{kHz})$ was inserted in the optical path and the transmitted signal was demodulated with a lock-in amplifier.

\section{Experiments in Tel21}

The Tel21 sample was prepared in $10 \mathrm{mM}$ sodium phosphate-buffer $(\mathrm{pH}=7)$ with 135 $\mathrm{mM} \mathrm{NaCl}$. As can be seen in fig. 5, the CD peak at $293 \mathrm{~nm}$ decreases with temperature, denoting the gradual loss of the $\mathrm{G} 4$ structure. A melting temperature of $62^{\circ} \mathrm{C}$ is inferred from these measurements. Fig. 6 displays the CD kinetics measured for 8 initial temperatures ranging from 21 to $73^{\circ} \mathrm{C}$ after a $8^{\circ} \mathrm{C}$ temperature jump. Measurements during the first second correspond to the steady-state CD and as expected, steady-state CD decreases with temperature. During the following 4 seconds, heating of the water is applied and one clearly sees that the CD drops, denoting the G4 unfolding. Then, temperature goes back to its initial value and the $\mathrm{CD}$ increases, demonstrating the reversibility of the unfolding process.

\section{Discussion}

Extraction of the relevant relaxation times in fig. 6 is unfortunately not straightforward because the observed dynamics are not very different from the solvent dynamics. We have therefore developed a deconvolution procedure to extract the G4 relaxation times. The principle is the following: from independent in situ measurement in a calibration sample (Phenyl Red), we know the exact temperature dynamics in the sample $T(t)$. From this information we can deduce the expected CD of the sample supposing that the G4 instantaneously follows the temperature change: $C D_{\text {instantaneous }}(T(t))$. This curve is depicted by the black lines in fig. 7 and one clearly sees that there is a time lag between this instantaneous curve and the experimental one. Introducing a relaxation 
time taking into account the folding/unfolding dynamics of the $\mathrm{G} 4\left(\tau_{G 4}\right)$, the evolution equation for the $\mathrm{G} 4 \mathrm{CD}$ reads:

$$
\frac{d C D}{d t}=-\frac{1}{\tau_{G 4}}\left(C D-C D_{\text {instantaneous }}(T(t))\right)
$$

Fitting of the experimental curves thanks to eq. (1) yields the real relaxation time of the G4s. The fits which are displayed as blue lines in fig. 7 allows us to extract times around $50 \mathrm{~ms}$ for the unfolding process (heating phase) and $70 \mathrm{~ms}$ for the folding process (cooling phase). As far as we know, those are the first measurements of the dynamics of the thermal folding/unfolding processes in Tel21. Those relaxation times are much larger than those observed for the peptide showing that the folding process at stake in G4 is much more complicated. The large interaction between guanines which offers many opportunities of misfolded structures could be responsible for such a feature [15].

\section{Conclusion}

Combination of T-jump and CD measurements is a powerful way to investigate rapid conformational changes in large biological molecules in solution. Two examples have been given: the folding/unfolding processes of $\alpha$-helices in PGA in a microsecond regime and of an antiparallel G4 structure (Tel21) in the millisecond one. Because it does not require any specific sample preparation or modification, this technique provides a powerful tool to investigate biological samples very close from their natural conditions.

Acknowledgements, The LABEX PALM (QUADfold and OSPEG projects, ANR-10-LABX0039-PALM) is gratefully acknowledged for financial support. 


\section{References}

[1] Burghardt, I.\& Haacke, S. eds. (2017). Ultrafast dynamics at the nanoscale Biomolecules and supramolecular assemblies. Pan Stanford Publishing, Singapore.

[2] Chergui, M. (2019). J. Chem. Phys., 150, 070901 (070915p).

[3] Fasman, G. D. e. (1996). Circular dichroism and the conformational analysis of biomolecules. Plenum Press, New York.

[4] Volk, M. (2001). European Journal of Organic Chemistry, 2001, 2605-2621.

[5] Callender, R. H.\& Dyer, R. B. (2002). Curr. Opin. Struct. Biol, 12, 628-633.

[6] Dobson, C. M. (2003). Nature, 426, 884-890.

[7] Review (2006). Chemical Review, 106, 1543-1977.

[8] Khuc, M. T., Mendonça, L., Sharma, S., Solinas, X., Volk, M.\& Hache, F. (2011). Rev. Sci. Instrum., 82, 054302 (054308p).

[9] Mendonça, L., Steinbacher, A., Bouganne, R.\& Hache, F. (2014). J. Phys. Chem. B, $118,5350-5356$.

[10] Spek, E. J., Gong, Y.\& Kallenbacj, N. R. (1995). J. Am. Chem. Soc., 117, 1077310774.

[11] Hänsel-Hertsch, R., Di Antonio, M.\& Balasubramanian, S. (2017). Nat. Rev., 18, 279-284.

[12] Lane, A. N., Chaires, J. B., Gray, R. D.\& Trent, J. O. (2008). Nucl. Acids Res., 36, $5482-5515$.

[13] Gray, R. D., Trent, J. O.\& Chaires, J. B. (2014). J. Mol. Biol., 426, 1629-1650.

[14] Schmid, M. (2017). PhD dissertation : Conformational dynamics of G-quadruplex DNA probed by time-resolved circular dichroism. Univ. Paris-Saclay.

[15] Stadlbauer, P., Mazzanti, L., Cragnolini, T., Wales, D. J., Derreumaux, P., Pasquali, S.\& Sponer, J. (2016). J. Chem. Theory Comput., 12, 6077-6097. 


\begin{tabular}{|l|l|l|l|l|}
\hline$p H$ & $\begin{array}{l}T_{m} \\
(K)\end{array}$ & $\begin{array}{l}E_{a, f} \\
(\mathrm{~kJ} / \mathrm{mol})\end{array}$ & $\begin{array}{l}E_{a, u} \\
(\mathrm{~kJ} / \mathrm{mol})\end{array}$ & $(\mathrm{kJ} / \mathrm{mol})$ \\
\hline 4.2 & $337 \pm 2$ & $21 \pm 4$ & $66 \pm 5$ & -45 \\
\hline 4.4 & $323 \pm 2$ & $11 \pm 2$ & $56 \pm 3$ & -45 \\
\hline 4.7 & $337 \pm 2$ & $7 \pm 3$ & $50 \pm 4$ & -43 \\
\hline 5.0 & $312 \pm 2$ & $6 \pm 5$ & $48 \pm 6$ & -42 \\
\hline 5.4 & $282 \pm 11$ & & & \\
\hline
\end{tabular}

Table 1. Activation energy for the folding $\left(E_{a, f}\right)$ and unfolding $\left(E_{a, u}\right)$ reactions extracted from the T-jump experiments (Ref. ).

\section{Figure captions}

Figure 1. Experimental T-jump/CD set-ups. (a) : nanosecond set-up with a probe at 220 nm; (b) millisecond set-up at $293 \mathrm{~nm}$.

Figure 2. Far-UV circular dichroism spectra of PGA (r.h.s : molecular structure) as a function of the temperature.

Figure 3. Absorption and CD dynamics at $220 \mathrm{~nm}$ in PGA after a $8^{\circ} \mathrm{C}$ T-jump.

Figure 4. Schematic of the energy profile of the $P G A_{\text {unfolded }}^{\stackrel{k_{f}}{\stackrel{\leftarrow}{k_{u}}}} P G A_{\text {folded }}$ reaction.

Figure 5. Far-UV circular dichroism spectra of Tel21 (r.h.s : scheme of an antiparallel G4 structure) as a function of the temperature.

Figure 6. Dynamics of CD a $293 \mathrm{~nm}$ in Tel 21 for a heating/cooling cycle.

Figure 7. Theoretical adjustment of the experimental data. Black curves: instantaneous response, blue curves: delayed G4 response, for the heating (a) and cooling (b) steps. 